\title{
Mathematical Model of Controlling the Spread of Malaria Disease Using Intervention Strategies
}

\author{
Fekadu Tadege Kobe \\ Department of Mathematics, College of Natural and Computational Science, Wachemo University, Hossana, Ethiopia \\ Email address: \\ feket200@gmail.com \\ To cite this article: \\ Fekadu Tadege Kobe. Mathematical Model of Controlling the Spread of Malaria Disease Using Intervention Strategies. Pure and Applied \\ Mathematics Journal. Vol. 9, No. 6, 2020, pp. 101-108. doi: 10.11648/j.pamj.20200906.11
}

Received: January 29, 2020; Accepted: March 9, 2020; Published: November 11, 2020

\begin{abstract}
This paper proposes and analyses a basic deterministic mathematical model to investigate Simulation for controlling the spread of malaria Diseases Transmission dynamics. The model has seven non-linear differential equations which describe the control of malaria with two state variables for mosquito's populations and five state variables for human's population. To represent the classification of human population we have included protection and treatment compartments to the basic SIR epidemic model and extended it to SPITR model and to introduce the new SPITR modified model by adding vaccination for the transmission dynamics of malaria with four time dependent control measures in Ethiopia Insecticide treated bed nets (ITNS), Treatments, Indoor Residual Spray (IRs) and Intermittent preventive treatment of malaria in pregnancy (IPTP). The models are analyzed qualitatively to determine criteria for control of a malaria transmission dynamics and are used to calculate the basic reproduction $\mathrm{R}_{0}$. The equilibria of malaria models are determined. In addition to having a disease-free equilibrium, which is globally asymptotically stable when the $\mathrm{R}_{0}<1$, the basic malaria model manifest one's possession of (a quality of) the phenomenon of backward bifurcation where a stable disease-free equilibrium co-exists (at the same time) with a stable endemic equilibrium for a certain range of associated reproduction number less than one. The results also designing the effects of some model parameters, the infection rate and biting rate. The numerical analysis and numerical simulation results of the model suggested that the most effective strategies for controlling or eradicating the spread of malaria were suggest using insecticide treated bed nets, indoor residual spraying, prompt effective diagnosis and treatment of infected individuals with vaccination is more effective for children.
\end{abstract}

Keywords: Malaria, Basic Reproduction Number, Backward Bifurcation Analysis, Vaccination and SPITR Modified Model

\section{Introduction}

Malaria is an infectious disease and is life threatening for human beings worldwide. Parasite is an organism that lives on or inside a human body from which it gets its food. Malaria is caused due to a parasite called Plasmodium. Plasmodium parasite is transmitted into human body when an infected female anopheles mosquito makes bites. Plasmodium parasites making the human liver as their home multiply their population and start infecting red blood cells of the human. A variety of plasmodium parasites exist. Mainly four types of plasmodium cause malaria disease among the human, falciparum, vivax, ovale and plasmodium malaria [1].

The plasmodium parasite is injected into the human bloodstream in the form or stage or life cycle known as sporozoite. The parasites go through a complex life cycle inside the hosting human body and they live at various stages both in liver and red blood cells. From time to time the parasites pass through various stages of their life cycle and during which numerous human red blood cells are destroyed.

The effect of malaria disease varies with the infecting variety species of Plasmodium and also with prior health and immune status of the individual. Typically malaria disease causes fever and chills together with headaches, vomiting and diarrhea. It may also cause long-term anemia, liver damage and neurological damage. The most dangerous falciparum parasite scan causes cerebral malaria. It causes frequently a fatal condition involving damaging the brain and central nervous system. The survived people from the cerebral malaria may too experience brain damage.

Malaria is a parasitic vector or mosquito borne disease. The malaria disease is found among particular people or in a 
particular region in many parts of the world [2]. Annually about 3 to 5 hundred million malaria cases are being identified worldwide and of which 1 to 3 million people end their lives with deaths [3].

About $40 \%$ of the world population lives in malaria endemic regions. The parasites and mosquitoes causing malaria have been developing their resistance powers against the drugs and chemicals that are being used to kill or destroy them. As a result, the incidents of malaria disease have been increasing in the past few decades.

In the recent time, significant resources and control programs have been made available worldwide. The aim is to reduce malaria infected cases and prevalence or gain upper hand over the disease. Different strategies and programs with varying effectiveness and efficiency are being adopted to control malaria disease. Comparative knowledge of these existing programs is necessary to design and organize any new and useful and cost effective procedure to control malaria epidemic $[4,5]$.

Efforts to reduce malaria transmission have led to the development of efficient vector control interventions, particularly insecticide treated nets (ITNs), indoor residual spraying (IRS), and larvicide [6, 7]. The ITNs include conventional nets treated with a WHO recommended insecticide and long-lasting insecticidal nets. Note that larva is an immature form of an insect and larvicide is a chemical used to kill larvae. These interventions are used in malaria endemic countries especially those in sub-Saharan Africa and have led to reduction in malaria morbidity and mortality substantially. However, malaria epidemic continues to claim hundreds of thousands of lives every year, thus necessitating a continued control effort to fight against the disease $[8,9]$.

Malaria has been considered as a global issue. Epidemiologists together with other scientists invest their efforts to understand the dynamics of malaria and to control transmission of the disease. From interactions with these scientists, mathematicians have developed tools called mathematical models. These models are used significantly and effectively for giving an insight into (i) the interaction between the host and vector population (ii) the dynamics of malaria disease (iii) control mechanisms of malaria transmission and (iv) effectiveness of eradication techniques.

Mathematical models are particularly helpful as they consider and include the relative effects of various sociological, biological and environmental factors on the spread of disease. The models have played a very important role in the development of malaria epidemiology. Analysis of mathematical models is important because they help in understanding the present and future spreads of malaria so that suitable control techniques can be adopted.

The SIR is a simplest mathematical model and has three classes or compartments, Susceptible, Infected and Recovered. Here we considered SIR model and modified it by adding two more compartments viz., protected and treatment classes. The inclusion of the protected and treatment classes to SIR model and extending it to SPITR model is a technique used to control the spread of malaria disease. Thus, we presented the SPITR model that describes the dynamics and controlling mechanism of malaria disease. The effect of controlling technique in the spread of malaria is analyzed [10]. This new model is an extension or modification of the existing mathematical models used to deal with malaria epidemic SIR, SEIR, SEIS, SEIIR [11-18]. The simulation studies of the model with variable values of sensitive parameter of the spread of malaria are performed and the results are incorporated. The necessary conclusions have been drawn.

\section{The SPITR Model}

Malaria disease occurs due to the bites of female anopheles mosquito. That is, the prevention and control rates of malaria disease will increase with the mortality or death rate of the female Anopheles mosquito. Hence, the need of inventing intervention strategies which will help to increase mortality of the female anopheles mosquitoes in addition to the natural mortality is arisen. In the recent times many intervention strategies are adopted worldwide. These intervention strategies increase the mortality rate of female anopheles mosquitoes and help directly to control the birth and spread of malaria. The important intervention strategies include (i) indoor residual spraying (IRS) and (ii) insecticide treated bed net (ITN). These intervention strategies play a significant role in the controlling mechanism of malaria disease.

\subsection{Classification of Human Compartments}

The present mathematical model is constructed with an objective of incorporating the effect of intervention strategies on the spread of malaria disease. Thus, we considered the protected and treatment class, added to SIR model and extended to SPITR model. In the SPITR model all the humans are divided into five classes or compartments and they exhibit the following properties:

Susceptible human class $\left(S_{h}\right)$ contains humans those do not have malaria disease but are likely to be bitten by infected female anopheles mosquitoes causing malaria. The people in this class do not take any protective measurements against malaria disease. In general, all the common people are included in this class. Humans of the susceptible class have the potential to remain either in the same class or to enter into protected class or infected class. People in this class are given awareness about malaria disease and advised to be protected using IRS and ITN mechanisms. The people who follow advises and adopt protected mechanisms will enter into protected class. The remaining people will remain in this class as long as they do not be bitten by infected female anopheles mosquitoes and enter into infected class.

Protected human class $(P)$ contains humans those do not have malaria disease. Also this class people are assumed that they will never get the disease. The people in this class do take some protective measurements like IRS and ITN and they become fully protected from malaria disease. These people keep themselves safe from malaria. The humans of this class are drawn from both susceptible and recovered classes.

Infected human class $\left(I_{h}\right)$ contains humans those are already infected and got malaria disease. People come into 
infected class from susceptible class. People from infected class have got the potential to enter into either treatment class or recovered class.

Treatment human class $(T)$ contains the humans those are already infected and got malaria disease. The people in this class are being given medical care or clinical treatment. People into the treatment class come from only infected class. After completion of the treatment successfully, the people from treatment class will go to recovered class.

Recovered human class $(R)$ contains the people who recover from the malaria disease andreturn to normal status of health. People come into the recovered class from both infected and treatment classes. People in this class are given awareness about malaria disease and advised to be protected using IRS and ITN mechanisms. The people who follow advises and adopt protected mechanisms will enter into protected class and the remaining people will go to susceptible class.

Further, we assume that the sick people do not travel far away or they go from place to place. Thus, we do not include the immigrations of malaria infected people in our model.

\subsection{Classification of Mosquito Compartments}

Anopheles is a mosquito of a genus which includes the species that transmits the malaria parasite to humans. Anopheles male mosquitoes do not carry plasmodium parasite carrying capacity and they do not cause malaria in humans. Hence, anopheles male mosquitoes are not included in the model. Infected female anopheles mosquitoes through bits cause malaria in humans. We have thus considered and included only female anopheles mosquito population in the present model. Further, note that only female anopheles mosquitoes bite humans for blood meals. In the present model all the female anopheles mosquitoes are divided into two classes or compartments and they exhibit the following properties:

Susceptible mosquito class $\left(S_{m}\right)$ contains the female anopheles mosquitoes. The mosquitoes in this class are not infected and their bites do not lead to malaria disease in humans. The population size of these mosquitoes grows with the natural birth rate of $\rho$ and diminishes with the natural death rate of $\omega$. The susceptible female anopheles mosquitoes get infected on feeding themselves with the blood of malaria - infected humans and then they leave susceptible mosquito class and enter infected mosquito class. Susceptible mosquitoes bite the infected humans with the rate of $\emptyset$. Further, it is assumed that the susceptible mosquitoes get infected with a rate of $\theta_{h m}$ when they bite infected humans.
Infected mosquito class $\left(I_{m}\right)$ containsthe infected female anopheles mosquitoes. The mosquitoes in this class are infected and their bites lead to malaria disease in humans. The population size of this class grows with a transfer rate proportional to $\alpha_{m}$ from the susceptible mosquito class. The infected mosquitoes diminish with the natural death rate of $\omega$. The infected female anopheles mosquitoes transfer infection to susceptible humans when the former feed them with the blood of the latter. Infected mosquitoes bite the susceptible humans with the rate of $\varnothing$. Further, it is assumed that the infected mosquitoes transfer infection with a rate of $\theta_{m h}$ when they bite susceptible humans.

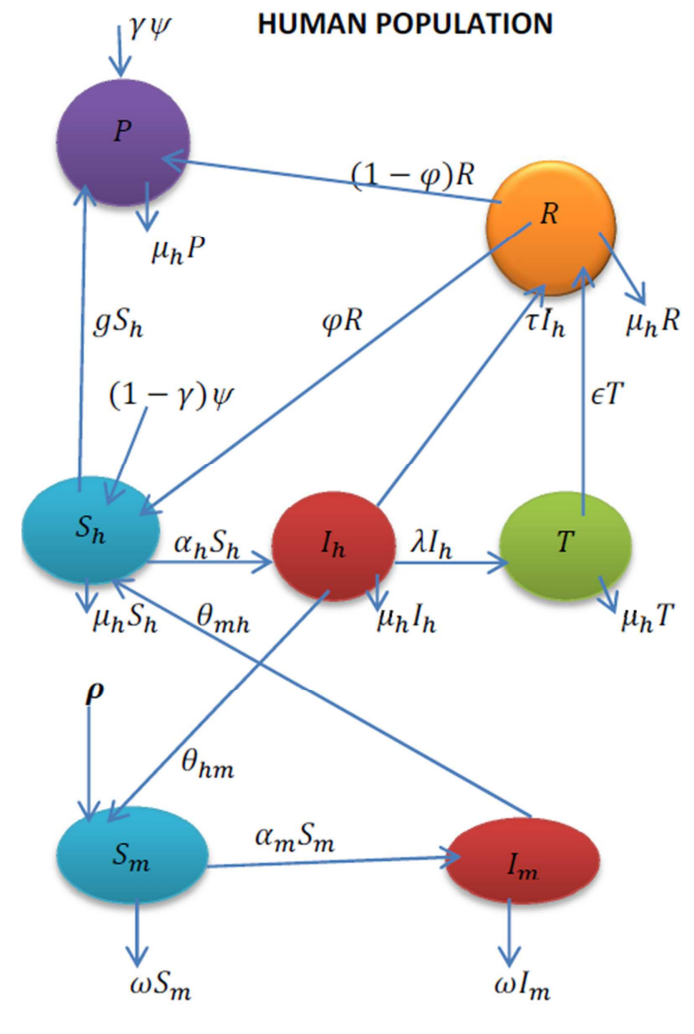

MOSUITO

\section{POPULATION}

Figure 1. Flow chart of SPITR malaria model.

The parameters in Table 1 and the state variables in Table 2 are used in Figure 1 to formulate the malaria model with control.

Table 1. Parameters of the model (1) and their interpretations.

\begin{tabular}{ll}
\hline Parameter & Description \\
\hline$\psi$ & Natural birth rate of humans \\
$\rho$ & Natural birth rate of mosquitoes \\
$\alpha_{h}$ & Transfer rate of humans from susceptible to infected compartment \\
$\alpha_{m}$ & Transfer rate of mosquitoes from susceptible to infected compartment \\
$\mu_{h}$ & Natural death rate for humans \\
$\delta_{h}$ & Death rate of humans due to malaria disease. This death rate in addition to the natural death rate is applicable to the humans of Infected \\
$\lambda$ & compartment. \\
$\epsilon$ & Transfer rate of humans from infected to treatment compartment \\
$\tau$ & Transfer rate of humans from treatment to recovered compartment \\
$\varphi$ & Transfer rate of humans from Infected to recovered compartment \\
$\theta_{m h}$ & Transfer rate of humans from recovered to susceptible compartment \\
\hline
\end{tabular}




\begin{tabular}{ll}
\hline Parameter & Description \\
\hline$\theta_{h m}$ & Probability of transmission of infection from an infectious human to a susceptible mosquito \\
$\gamma$ & Fraction of natural birth rate of humans. Newly born humans enter into susceptible and protected compartments with the rates of(1 - \\
$g$ & $\gamma) \psi$ and $\gamma \psi$ respectively. \\
$\omega$ & Transfer rate of humans from susceptible to protected compartment \\
$\emptyset$ & Death rate of mosquitoes. Natural deaths and deaths due to chemicals and other reasons are all included. \\
& Susceptible mosquitoes bite infected humans with this rate. \\
\hline
\end{tabular}

Table 2. Variables of the model (1) and their interpretations.

\begin{tabular}{ll}
\hline Variables & Description \\
\hline$S_{h}(t)$ & Number of humans insusceptible compartment at time $t$ \\
$P(t)$ & Number of humans in protected compartment at time $t$ \\
$I_{h}(t)$ & Number of humans in infected compartment at time $t$ \\
$T(t)$ & Number of humans in treatment compartment at time $t$ \\
$R(t)$ & Number of humans in recovered compartment at time $t$ \\
$S_{m}(t)$ & Number of mosquitoes in susceptible compartment at time $t$ \\
$I_{m}(t)$ & Number of mosquitoes in infected compartment at time $t$ \\
$N_{h}(\mathrm{t})$ & Total human population at time $t$ \\
$N_{m}(\mathrm{t})$ & Total mosquito population at time $t$ \\
\hline
\end{tabular}

\subsection{Mathematical Formulation of SPITR Model}

Applying the assumptions, definitions of compartmental variables and parameters described in tables 1 and 2, the system of non-linear differential equations which describe the dynamics of malaria transmission with controlling measures are formulated and presented in this section.

$$
\begin{gathered}
\mathrm{dS}_{h} / d t=(1-\gamma) \psi-\frac{\theta_{h m} \emptyset \mathrm{I}_{\mathrm{m}}}{N_{h}} S_{h}-\mu_{h} S_{h}-g S_{h}+\varphi R \\
d \mathrm{P} / d t=\gamma \psi+g S_{h}+(1-\varphi) R-\mu_{h} P \\
d \mathrm{I}_{h} / \mathrm{dt}=\frac{\theta_{h m} \emptyset \mathrm{I}_{\mathrm{m}}}{N_{h}} S_{h}-\tau \mathrm{I}_{h}-\lambda \mathrm{I}_{h}-\left(\mu_{h}+\delta_{h}\right) \mathrm{I}_{h} \\
d T / d t=\lambda \mathrm{I}_{h}-\epsilon T-\mu_{h} T
\end{gathered}
$$

$$
\begin{gathered}
d \mathrm{R} / \mathrm{dt}=\epsilon \mathrm{T}+\tau \mathrm{I}_{\mathrm{h}}-(1-\varphi) \mathrm{R}-\varphi \mathrm{R}-\mu_{\mathrm{h}} \mathrm{R} \\
d S_{m} / d t=\rho-\frac{\theta_{h m} \emptyset \mathrm{I}_{\mathrm{h}}}{N_{h}} S_{m}-\omega S_{m} \\
d \mathrm{I}_{m} / d t=\frac{\theta_{h m} \emptyset \mathrm{I}_{\mathrm{h}}}{N_{h}} S_{m}-\omega \mathrm{I}_{m}
\end{gathered}
$$

\subsection{Proposed Modified Model}

We assumed that susceptible and infected mosquitoes are killed at same rate by humans and the inclusion of a vaccination parameter gives the modified SPITR model below. Again applying the assumptions, definitions of compartmental variables and parameters described in tables 1 and 2 , the system of non-linear differential equations which describe the dynamics of malaria transmission with controlling measures are formulated and presented in this section.

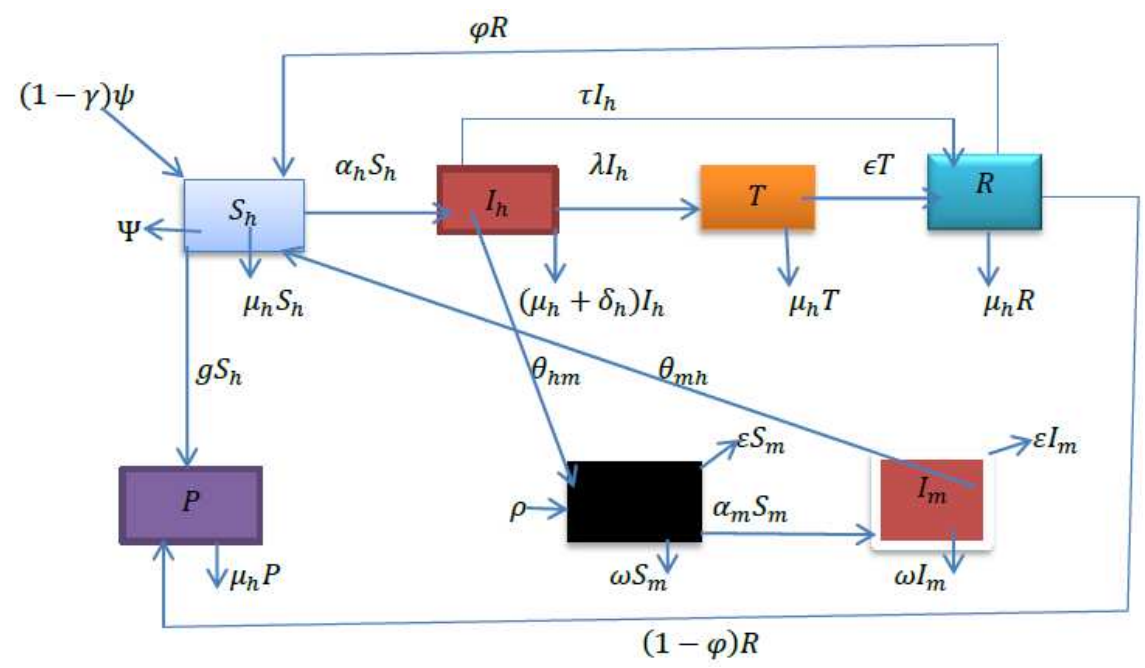

Figure 2. Flow chart of SPITR malaria modified model.

$$
\begin{gathered}
\mathrm{dS}_{h} / d t=(1-\gamma) \psi-\frac{\theta_{h m} \emptyset \mathrm{I}_{\mathrm{m}}}{N_{h}} S_{h}-\mu_{h} S_{h}-g S_{h}+\varphi R-\Psi S_{h} \\
d \mathrm{P} / d t=\gamma \psi+g S_{h}+(1-\varphi) R-\mu_{h} P \quad \text { (9) } \quad d \mathrm{I}_{h} / \mathrm{dt}=\frac{\theta_{h m} \emptyset \mathrm{I}_{\mathrm{m}}}{N_{h}} S_{h}-\tau \mathrm{I}_{h}-\lambda \mathrm{I}_{h}-\left(\mu_{h}+\delta_{h}\right) \mathrm{I}_{h}
\end{gathered}
$$




$$
\begin{gathered}
d T / d t=\lambda \mathrm{I}_{h}-\epsilon T-\mu_{h} T \\
d \mathrm{R} / \mathrm{dt}=\epsilon \mathrm{T}+\tau \mathrm{I}_{\mathrm{h}}-(1-\varphi) \mathrm{R}-\varphi \mathrm{R}-\mu_{\mathrm{h}} \mathrm{R} \\
d S_{m} / d t=\rho-\frac{\theta_{h m} \varnothing \mathrm{I}_{\mathrm{h}}}{N_{h}} S_{m}-\omega S_{m}-\varepsilon S_{m} \\
d \mathrm{I}_{m} / d t=\frac{\theta_{h m} \emptyset \mathrm{I}_{\mathrm{h}}}{N_{h}} S_{m}-\omega \mathrm{I}_{m}-\varepsilon I_{m}
\end{gathered}
$$

The initial conditions of the system of equations (1) are given by $S_{h}(0)=S_{h 0}, \mathrm{P}(0)=\mathrm{P}_{0}, I_{h}(0)=I_{h 0}, \mathrm{~T}(0)=$ $\mathrm{T}_{0}, \mathrm{R}(0)=\mathrm{R}_{0}, S_{m}(0)=S_{m 0}$ and $I_{m}(0)=I_{m 0}$. Also, we have used in equation (1) that $\alpha_{h}=\left(\theta_{m h} \varnothing \mathrm{I}_{\mathrm{m}} / N_{h}\right)$ and $\alpha_{m}=\left(\theta_{h m} \varnothing \mathrm{I}_{\mathrm{h}} / N_{h}\right)$. The term $\alpha_{h}$ denotes the rate at which the susceptible humans become infected by infectious mosquitoes. Similarly, the term $\alpha_{m}$ denotes the rate at which the susceptible mosquitoes become infected by infectious humans. The rate of infection propagated to susceptible humans by infected mosquitoes is dependent on the total number of humans. Similarly, the rate of infection propagated to susceptible mosquitoes by infected humans is dependent on the total number of humans.

\section{Analysis of SPITR Model}

We now analyze the SPITR model in order to show the two controlling methods considered here have substantial impact on controlling the spread of malaria disease. In fact, the disease will be completely eradicated if the controlling methods are implemented effectively. The two controlling mechanisms proposed here have such a big potential. We consider now the solutions of the system of non-linear differential equation (1). We understand that the interpretations of these solutions must be biologically meaningful. Hence it is easy to identify that the feasible region of system (1) is $\mathbb{R}_{+}^{7}$. The seven dimensional solution space shows that all the solutions are positive. Hence, the feasible region containing all the solutions of the system of equations (1) is given by the set $\Omega=\left\{\left(S_{h}, P, I_{h}, T, R, S_{m}, I_{m}\right) \in \mathbb{R}_{+}^{7}\right\}$. Here the quantities $S_{h}, P, I_{h}, T, R, S_{m}, I_{m}$ are all non - negatives. Further the total human and mosquito populations are represented by $N_{h}$ and $N_{m}$ they have the upper asymptotic values $\left(\psi / \mu_{h}\right)$ and $(\rho / \omega)$ respectively. Therefore, the region $\Omega$ is positively invariant i.e. solutions remain positive for all the temporal values. Thus, the model (1) is biologically meaningful and mathematical well-posed or well present in the domain $\Omega$.

On summing up all the individual equations from (1a) to (1e) of the system (1), it is straight forward to get $\left(d N_{h} / d t\right)=$ $\left(\psi-\mu_{h} N_{h}-\delta_{h} \mathrm{I}_{h}\right)$. Here the notation $N_{h}=\left(S_{h}+P+I_{h}+\right.$ $T+R)$ represents the total human population contained in all the five compartments. We consider the solution of the system of equations (1) when the term $\delta_{h} \mathrm{I}_{h}$ vanishes. In case if the death rate of humans due to malaria disease is considered to be free, i.e., $\delta_{h}=0$ then we obtain $\left(d N_{h} / d t\right)=\left(\psi-\mu_{h} N_{h}\right)$. The solution of this differential equation is found to be $N_{h}(t)=\left(\psi / \mu_{h}\right)+\left[N_{h 0}-\left(\psi / \mu_{h}\right)\right] e^{-\mu_{h} t}$ showing that $N_{h}(t) \rightarrow \psi / \mu_{h}$ as $t \rightarrow \infty$. The term $N_{h 0}$ denotes the initial total human population. It can be interpreted that the total human population grows and asymptotically converges to a positive quantity given by $\left(\psi / \mu_{h}\right)$ under the condition that humans do not die due to malaria infection. Thus $\psi / \mu_{h}$ is an upper bound of the total human population $N_{h}(t)$ i.e. $N_{h}(\infty) \leq$ $\psi / \mu_{h}$. Whenever the initial human population starts off low below $\left(\psi / \mu_{h}\right)$ then it grows over time and finally reaches the upper asymptotic value $\left(\psi / \mu_{h}\right)$. Similarly, whenever the initial human population starts off high above $\left(\psi / \mu_{h}\right)$ then it decays over time and finally reaches the lower asymptotic value $\left(\psi / \mu_{h}\right)[19]$.

Similarly on summing up all the individual equations from (1f) to (1g) of the system (1), it is straight forward to get $d N_{m} / d t=\rho-\omega N_{m}$. Here the notation $N_{m}=\left(S_{m}+I_{m}\right)$ represents the total mosquito population contained in all the two compartments. The solution of this differential equation is found to be $N_{m}(t)=(\rho / \omega)+\left[N_{m 0}-(\rho / \omega)\right] e^{-\omega t}$ showing that $N_{m}(t) \rightarrow(\rho / \omega)$ as $t \rightarrow \infty$. The term $N_{m 0}$ denotes the initial total mosquito population. It can be interpreted that the total mosquito population grows and asymptotically converges to a positive quantity given by $(\rho / \omega)$. Thus $(\rho / \omega)$ is an upper bound of the total mosquito population $N_{m}(t)$ i.e. $N_{m}(\infty) \leq(\rho / \omega)$. Whenever the initial mosquito population starts off low below $(\rho / \omega)$ then it grows over time and finally reaches the upper asymptotic value $(\rho / \omega)$. Similarly, whenever the initial mosquito population starts off high above $(\rho / \omega)$ then it decays over time and finally reaches the lower asymptotic value $(\rho / \omega)$.

\subsection{Disease Free Equilibrium Point}

Disease free equilibrium points are steady state solutions when there is no malaria in the human population and there is no plasmodium parasite in the mosquito population. That is, absence of malaria causing infections occurs in both populations at the disease free equilibrium point. The disease free equilibrium point is denoted by $E_{0}=\left(S_{h}^{*}, I_{h}^{*}, P^{*}, T^{*}, R^{*}, S_{m}^{*}, I_{m}^{*}\right)$. The equilibrium point is obtained on setting the right-hand side of the non-linear system (1) to zero. Thus, at the equilibrium point the quantities satisfy the condition $I_{h}^{*}=P^{*}=T^{*}=R^{*}=I_{m}^{*}=$ $0, S_{h}^{*}=\left(\psi / \mu_{h}\right)$ and $S_{m}^{*}=(\rho / \omega)$. Also, Overhead star represents the values of the functions at the disease free equilibrium point. The disease free equilibrium point represents $E_{0}$ the disease free situation in which there is no malaria infection either in the society or in the environment. Therefore, the diseases free equilibrium point is given by

$$
E_{0}=\left(\psi / \mu_{h}, 0,0,0,0, \rho / \omega, 0\right)
$$

\subsection{Basic Reproduction Number $R_{0}$}

Reproduction number, denoted by $R_{o}$, is the threshold or a level for many epidemiological models. It determines whether a disease can attack the population or not. The threshold quantity $R_{0}$ indicates the number of new infected individuals is produced by one infected individual. When $R_{0}<0$ each infected individual propagates the infection and produces on average less than one new infected individual so that the disease is expected to die out completely over time. On the other hand if $R_{0}>1$, each individual produces more 
than one new infected individual so we would expect the disease to spread more and grow in the population. This means that the value of threshold quantity $R_{0}$ in order to eradicate the disease must be reduced by less than one. We determine the expression for $R_{0}$ using the next generation matrix approach [19-21] as $R_{0}=\sqrt{\frac{\rho \theta_{m h} \theta_{h m} \emptyset^{2} \mu_{h}}{\omega^{2} \psi\left(\tau+\lambda+\mu_{h}+\delta_{h}\right)}}$. Further, it can be verified that the disease free equilibrium point $E_{0}$ given by (4) is locally asymptotically stable if $R_{0}<1$ and unstable if $R_{0}>1$.

\section{Numerical Simulation}

In this section we consider the simulation study of the system of differential equations given in (1). As stated earlier these equations describe the dynamics of human and mosquito populations of the malaria model that includes intervention strategies. The simulation study is performed using ode45 solver of MATLAB software. The Runge Kutta fourth-order method based on a variable step-size is used for the purpose. The parametric values have been collected from the literature and used here. The estimated parameters for the model (1) have been collected from the literature [22-28] and used here for the purpose of simulation study. They are $\lambda=0.01, €=0.00722, \gamma=0.11, g=0.86$, $\delta_{h}=0.0000027, \quad \mu_{h}=1 / 70 \times 365.25, \quad \theta_{h m}=0.0655$, $\varphi=1 / 20 \times 365.25, \quad \omega=1 / 25, \quad \varnothing=0.40, \quad \theta_{m h}=0.42$, $\psi=0.00051, \rho=0.0071$ and $\tau=1 / 7$. The rates of these parameters used here are taken per day.

We simulated the malaria model with the intervention strategies included to describe the dynamics of the human compartments with varied parametric values. In all the simulation studies performed here we fixed the initial values of the parameters and used as $S_{h 0}=57186, P_{0}=800, I_{h o}=$ $47473, T_{0}=3300, \quad R_{0}=37470, \quad S_{m 0}=18500$ and $I_{m 0}=36250$. We also compare the protected and treated classes in order to observe the impact of the interventions on the spread control of malaria. Here we consider two intervention strategies viz. INT and IRS, as controlling measures of malaria.

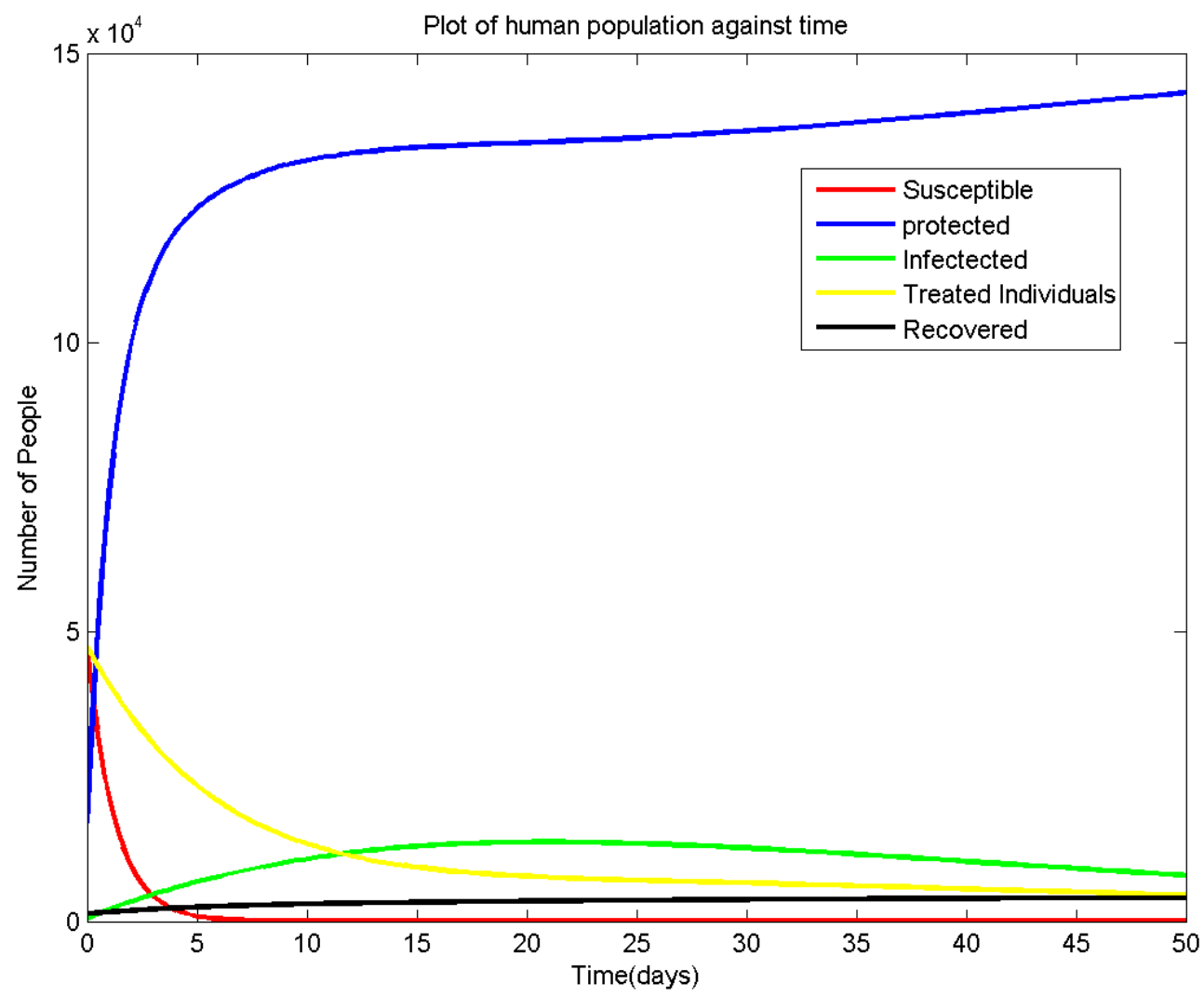

Figure 3. Dynamics of population sizes of human compartments $R_{0}=0.8741$.

Figure 3 shows the comparative population sizes of five human compartments. Here we have considered two intervention strategies viz. INTs and IRS to protect the human individuals of the protected compartment. The situation in the figure 1 corresponds to the reproductive ratio $R_{0}=0.8741$. That is, if the intervention strategies are implemented to that order so as to reduce $R_{0}$ to a value equivalent to 0.8741 the situation shown in this figure occurs. As a result (i) the population size of the protected compartment increases, (ii) the population sizes of susceptible, treated and recovered compartments decrease and (iii) the population size of the infected class rises slightly for some time and then falls. Further, the comparative study promises that the filing of protected compartment leads to emptying the remaining compartments and nullifying the malaria disease among the population. The control measures ITNs and IRS reduce the availability of hosts and kill mosquitoes. The spread of malaria disease is reduced as the mosquitoes that are attempting to feed themselves from human hosts is reduced. There is an increase in the infection 
when the protection is low and decrease in the infection when the protection is high. That is, the infection and protection are inversely proportional to each other. The two preventive measures recommended here to protect humans from mosquitoes are effective and economical and hence can be implemented. The netting also acts as a protective access to against bites, making it an ideal prevention method in low level areas where mosquitoes are found more in size.

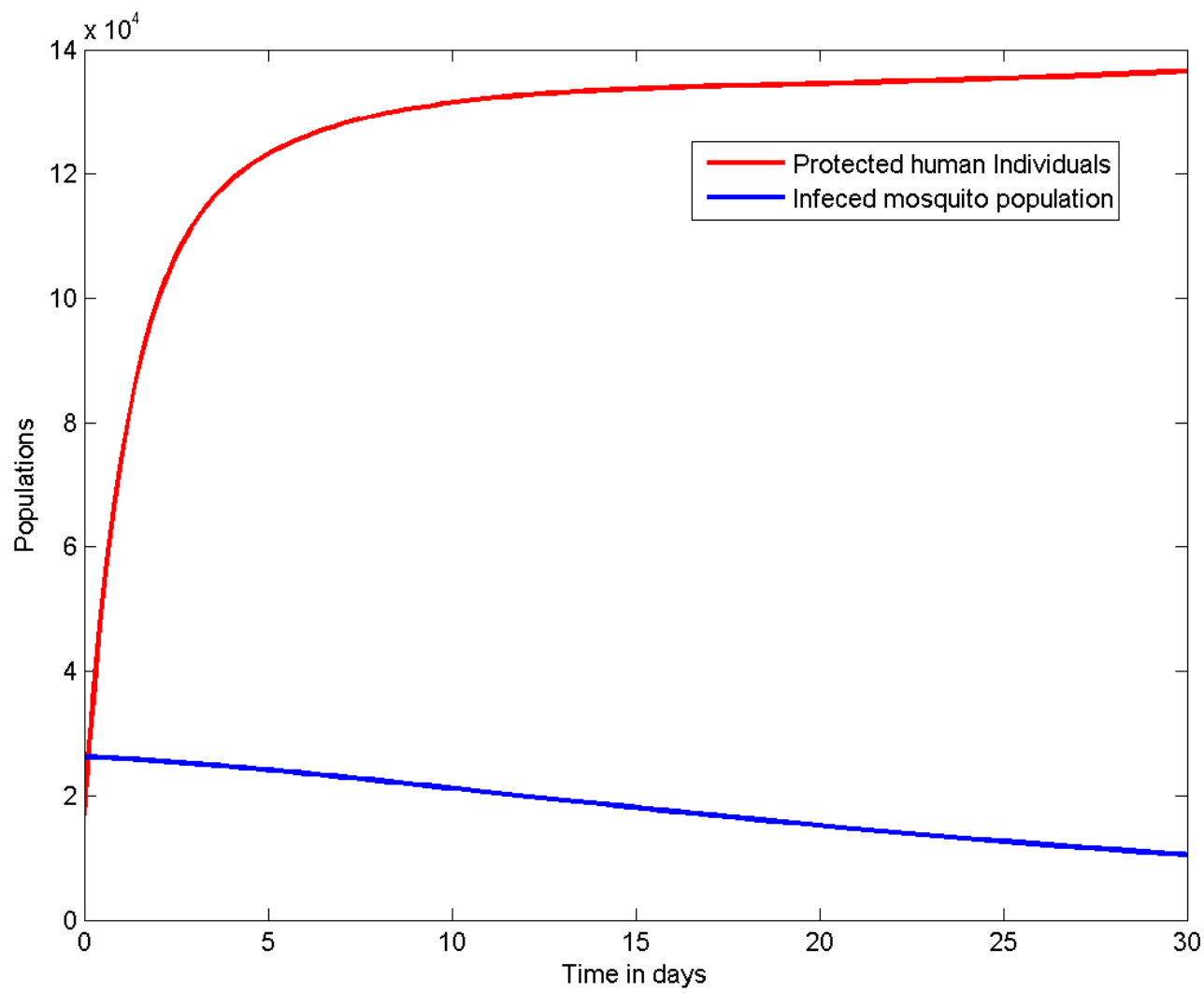

Figure 4. Comparative population dynamics of protected human and infected mosquito compartments when $R_{0}=0.8741$.

Figure 4 shows the comparative population dynamics of protected human and infected mosquito compartments. The population sizes of protected human and infected mosquito compartments are proportional to each other. If the protected population size of humans increased using intervention techniques then the infected mosquitoes size is decreased and vice versa.

\section{Conclusions}

We presented and analyzed a basic deterministic mathematical model to understand better how to control the spread of malaria. Then protected and treated classes were added to the basic SIR model and extended it to SPITR mode. The protected class is due to the introduction of the two intervention strategies ITNs and IRS. The potential impact of protection and treatment strategies on the transmission of malaria disease are considered. The present model includes the protection features those are effective to control the transmission of malaria disease and used worldwide.

The numerical analysis of the model suggested that effective control or eradication of malaria can be achieved by the combination of protection and treatment measures. We have that three intervention strategies are combined; there is a greater reduction in the number of infected individuals. The prevention strategies played a greater role in reducing the number of infected individuals by lowering the contact rate between the mosquito and human populations, for instance, through the use of ITNs. On the other hand both prevention strategies led to the reduction of the mosquito population hence lowering the infected mosquito population. Effective treatment consolidated the prevention strategies. This paper provides useful tools for assessing the effectiveness analysis of a combination of the intervention strategies and analyzing the potential impact of prevention with treatment.

\section{References}

[1] WHO (2009), "Global Malaria Program: Position Statement on ITNs".

[2] Nita. H. Shah and Jyoti Gupta (2013),"SEIR Model and Simulation for Vector Borne Diseases", Applied Mathematics Vol. 4, Pp. 13-17.

[3] Samwel Oseko Nyachae, Johana K. Sigey Jeconiah A. Okello, James M. Okwoyo and D. Theuri (2014),"A Study for the Spread of Malaria in Nyamira Town - Kenya, The SIJ Transactions on Computer Science Engineering and its Applications (CSEA), The Standard International Journals (The SIJ), Vol. 2, No. 3 (1), Pp. 53-60. 
[4] Rollback Malaria. What is malaria? http://www.rollbackmalaria.pdf. (2010-05-10).

[5] WHO (2007) Insecticide treated mosquito nets: a WHO position statement. Technical report, World Health Organization.

[6] Bayoh MN, Mathias DK, Odiere MR, Mutuku FM, Kamau L, et al.. (2010) Anopheles gambiae: historical population decline associated with regional distribution of insecticidetreated bed nets in western Nyanza province, Kenya. Malaria Journal 9.

[7] WHO (2004) Global strategic framework for integrated vector management. Technical report, World Health Organization, Geneva.

[8] WHO (2011) World Malaria Report. Technical report, World Health Organization, Geneva.

[9] RBM (2013) Minutes of roll back malaria vector control working group 8th annual meeting. Technical report, Roll Back Malaria. Available: http://www.rollbackmalaria.org/mechanisms/vcwg.html.

[10] P. M. M. Mwamtobe (2010), "Modeling the Effects of Multi Intervention Campaigns for the Malaria Epidemic in Malawi", M. Sc. (Mathematical Modeling) Dissertation, University of Dar es Salaam, Tanzania.

[11] G. A. Ngwa, W. S. Shu, A mathematical model for endemic malaria with variable human and mosquito populations. Mathematical and computer modeling, 32 (7), (2000), 747 763.

[12] C. Chiyaka J. M. Tchuenche, W. Garira \& S. Dube, A mathematical analysis of the effects of control strategies on the transmission dynamics of malaria. Applied Mathematics and Computation, 195 (2), (2008), 641-662.

[13] N. Chitnis J. M. Cushing \& J. M. Hyman, Bifurcation analysis of a mathematical model for malaria transmission. SIAM Journal on Applied Mathematics, 67 (1), (2006), 24-45.

[14] A. M. Baba, A Mathematical Model for Assessing the Control of and Eradication strategies for Malaria in a Community, Science.pub.net 4 (2), (2012), 7-12.

[15] Kermack WO, McKendrick AG (August 1, 1927). "A Contribution to the Mathematical Theory of Epidemics". Proceedings of the Royal Society A 115 (772): 700-721. doi: 10.1098/rspa.1927.0118.
[16] Bartlett MS (1957). "Measles periodicity and community size". Journal of the Royal Statistical Society, Series A 120 (1): 4870. Doi: 10.2307/2342553. JSTOR 2342553.

[17] Dejen Ketema Mamo and Purnachandra Rao Koya. "Mathematical Modeling and Simulation Study of SEIR disease and Data Fitting of Ebola Epidemic spreading in West Africa" Journal of Multidisciplinary Engineering Science and Technology (JMEST) Vol. 2 Issue 1, January 2015, pp 106 - 14. http://www.jmest.org/wp-content/uploads/JMESTN42350340.pdf.

[18] Purnachandra Rao Koya and Dejen Ketema Mamo, Ebola Epidemic Disease: Modelling, Stability Analysis, Spread Control Technique, Simulation Study and Data Fitting, Journal of Multidisciplinary Engineering Science and Technology (JMEST), Vol. 2, Issue 3, March 2015, pp 476 - 84 http://www.jmest.org/wp-content/uploads/JMESTN42350548.pdf.

[19] O. J. Diekmann (1990), "Mathematical Biology" Vol. 28, Pp. 365-382.

[20] Birkhoff G and Rota, G. C (1982), Ordinary Differential Equations, Ginn.

[21] Namawejje H. (2011). Modelling the Effect of Stress on the Dynamics and Treatment of Tuberculosis. M. Sc. (Mathematical Modelling) Dissertation, University of Dares Salaam, Tanzania.

[22] L. Perko, Differential Equations and Dynamics Systems in Applied Mathematics, vol. 7, Springer, Berlin, Germany, 2000.

[23] WHO/World Malaria Report (2014) (www.who.int/malaria).

[24] Chitnis N. (2005), "Using Mathematical Models in Controlling the Spread of Malaria", Ph. D. Thesis, Program in Applied Mathematics, University of Arizona, Tucson.

[25] Niger A shrafi M and Gumel Abba B (2008,"Mathematical Analysis of the Role of Repeated Exposure on Malaria Transmission Dynamics", Differential Equations and Dynamical Systems, Volume 16, No. 3, pp 251-287.

[26] Flores J. D. (2011). Math-735: Mathematical Modelling, 4.5 Routh-Hurwitz criteria. Department of Mathematical Sciences, The University of South Dakot.

[27] Y. T. Tumwiine, Mugisha and L. S. Lubobi (2007), "Applied Mathematics and Computation", Vol. 189, Pp. 1953-1965.

[28] Federal Democratic Republic of Ethiopia Ministry Of Health Ethiopia National Malaria Indicator Survey, Addis Ababa, 2008 . 